\title{
Concurrent Primary Follicular Lymphoma and a Mature Cystic Teratoma of the Ovary: A Case Report and Review of Literature
}

\author{
Sakara Hutspardol $\mathbb{D}^{1,2}$ Yi Li, $^{3,4}$ Valerie Dube, ${ }^{3,4}$ and Jan Delabie ${ }^{4,5}$ \\ ${ }^{1}$ Department of Pathology, Vancouver General Hospital, Vancouver, British Columbia, Canada \\ ${ }^{2}$ Department of Pathology and Laboratory Medicine, University of British Columbia, Vancouver, British Columbia, Canada \\ ${ }^{3}$ Trillium Health Partners/Credit Valley Hospital, Mississauga, Ontario, Canada \\ ${ }^{4}$ Department of Laboratory Medicine and Pathobiology, University of Toronto, Toronto, Ontario, Canada \\ ${ }^{5}$ Laboratory Medicine Program, University Health Network and University of Toronto, Toronto, Ontario, Canada \\ Correspondence should be addressed to Sakara Hutspardol; sakara.hutspardol@vch.ca
}

Received 24 October 2021; Accepted 11 February 2022; Published 25 February 2022

Academic Editor: George Adonakis

Copyright (c) 2022 Sakara Hutspardol et al. This is an open access article distributed under the Creative Commons Attribution License, which permits unrestricted use, distribution, and reproduction in any medium, provided the original work is properly cited.

\begin{abstract}
Primary lymphoma concurrent with teratoma of the ovary is exceedingly rare. Based on our review of the literature, there are only 8 case reports describing concurrent primary diffuse large B-cell lymphoma and teratoma. Here, we report the first case of primary follicular lymphoma concurrent with mature ovarian cystic teratoma, which, to our knowledge, has not been described in the literature.
\end{abstract}

\section{Introduction}

Mature cystic teratomas are the commonest ovarian tumor, accounting for $20 \%$ of all ovarian neoplasms and up to $60 \%$ of benign ovarian tumors during the reproductive years [1]. However, some tumors are not detected until postmenopause [2].

Most ovarian lymphomas represent secondary involvement by a systemic disease [3]. In comparison, primary ovarian lymphomas are rare and account for less than $1 \%$ of all cases of non-Hodgkin's lymphomas (NHLs) [4]. Concurrent involvement by NHL and MCT is exceedingly rare with only eight reported cases in the literature (Table 1). In all cases, diffuse large B-cell lymphoma was the lymphoma type.

Here, we report the occurrence of primary Stage IE, Grade 3A ovarian follicular lymphoma within a MCT in a 62 -year-old woman who underwent a laparoscopic bilateral salpingo-oophorectomy and omentectomy. Thorough radiologic investigations and staging bone marrow studies excluded a systemic disease. To our knowledge, this is the first case report of primary follicular lymphoma concurrent with MCT. The exclusive presence of follicular lymphoma within the MCT suggests it may have arisen from it.

\section{Case Report}

A 62-year-old female presented with pelvic discomfort without constitutional symptoms. CT abdomen demonstrated a $15.1 \times 12.2 \times 16.3 \mathrm{~cm}$ right ovarian solid and cystic mass with minimal calcification. Laboratory investigation reveals minimally elevated CA- 125 of 55 (normal $<35 \mathrm{U} / \mathrm{mL}$ ) and CA 19-9 less than 3 (normal $<27 \mathrm{kU} / \mathrm{L}$ ). Physical examination showed a palpable right lower quadrant abdominal mass without lymphadenopathy.

The patient underwent a laparoscopic bilateral salpingooophorectomy, omentectomy, and right ureterolysis. A $15 \mathrm{~cm}$ diameter right solid adnexal mass attached to the pelvic wall was excised with rupture of a cystic component.

Histological examination revealed fragments of benign squamous epithelium, ciliated respiratory-type epithelium, and hair component-associated foreign-body type granulomatous reaction. There were also cystic areas which lined by the squamous epithelium. The overall appearance was 


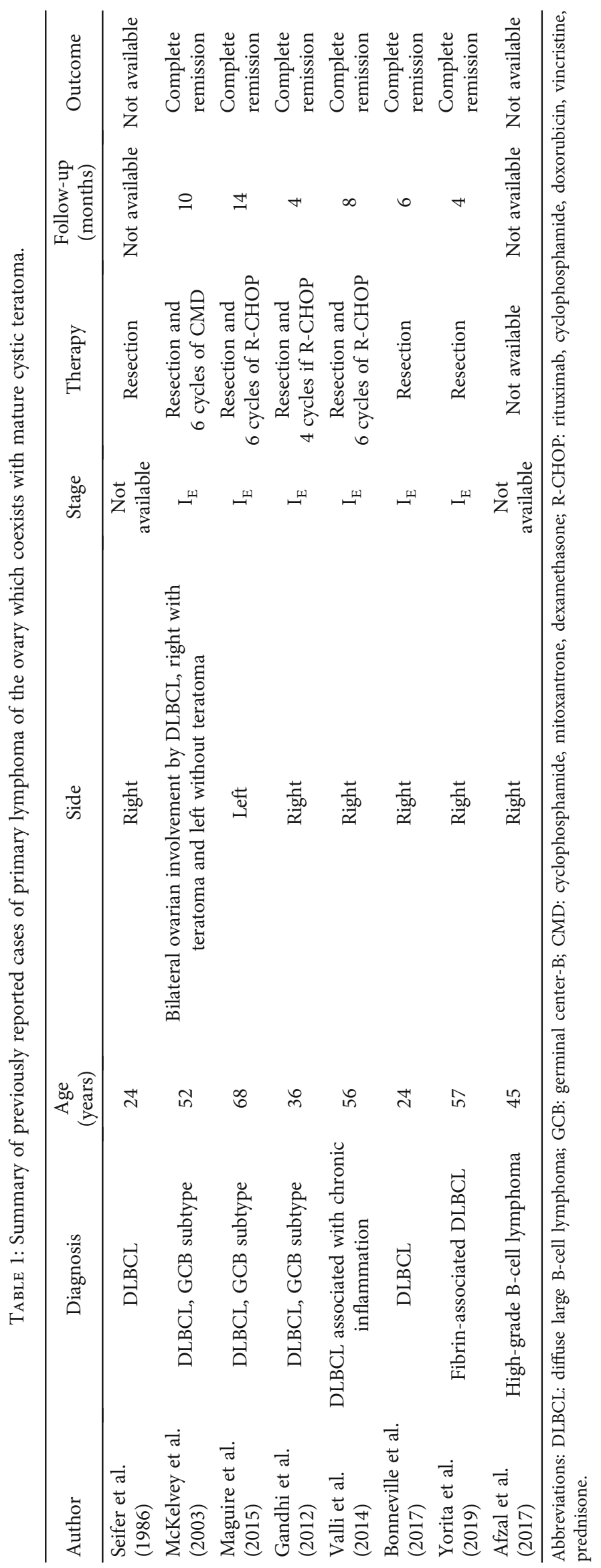




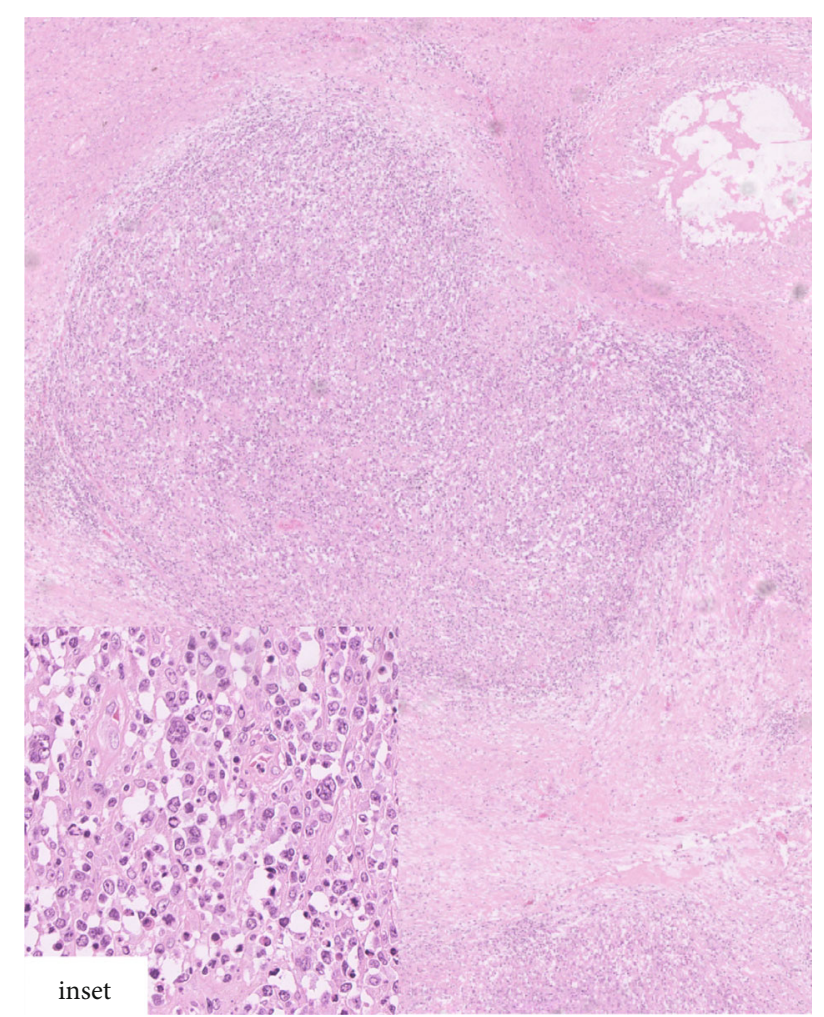

Figure 1: Hematoxylin and eosin staining ( $\mathrm{H}$ \& E 200x). Composite histology of follicular lymphoma within the mature cystic teratoma. The inset shows high-power view of the follicular lymphoma component comprising predominantly centroblasts and scattered centrocytes (H \& E 400x).

consistent with a mature cystic teratoma.

Further microscopic examination revealed very focal nodular infiltrates of atypical lymphoid cells within the teratoma (Figure 1). These lymphoid aggregates were composed primarily of intermediate to large-sized atypical cells with irregular nuclei, open chromatin, distinct nucleoli, and abundant cytoplasm, morphologically consistent with predominantly atypical small and large centroblasts (more than 15 per high power field). Scattered small centrocytes were also seen. Focal necrosis was seen in some of the aggregates.

Immunoperoxidase studies showed that the atypical cells are B lymphocytes with germinal center cell immunophenotype (expressing CD10 and BCL6) and showed aberrant expression of BCL2 (weak). They are associated CD21positive follicular dendritic cell meshworks. The Ki-67 proliferation index is $80 \%$ in the neoplastic cells. The overall morphologic and immunohistochemical findings are consistent with a grade $3 \mathrm{~A}$ follicular lymphoma (Figures 2(a)-2(f)).

The patient underwent further staging investigations, including a whole-body PET scan and CT scans of the head and neck, chest, abdomen, and pelvis, which show no evi- dence of systemic lymphoma. A bilateral bone marrow biopsy showed no bone marrow involvement. Therefore, the follicular lymphoma is primary to the ovary and arises as a part of the mature teratoma.

The patient was followed postoperatively every 3 months without further treatment and remained in complete clinical and radiological remission 18 months after the diagnosis.

\section{Discussion}

Mature cystic teratoma (MCT) is the most common germ cell neoplasm of the ovary in females of reproductive age. The majority of cases, particularly in younger women, are benign and treated with resection alone. In contrast, periand postmenopausal women with MCT have a higher risk of malignant transformation at approximately $1-2 \%$ with squamous cell carcinoma being the most common type of malignancy accounting for $80-90 \%$ of cases [5].

Primary lymphoma as a neoplastic component of ovarian teratoma is exceptionally rare with only 8 reported cases in the literature (Table 1); the lymphoma type reported in all cases is diffuse large B-cell lymphoma. Of the eight patients, five presented with localized unilateral disease, and one presented with bilateral ovarian involvement. The remaining two patients do not have complete staging investigation [6, 7]. Three patients were given R-CHOP chemotherapy and one given cyclophosphamide, mitoxantrone, and dexamethasone. All four patients who received postoperative adjuvant chemotherapy showed no evidence of disease recurrence upon follow-up [8-11]. Two patients underwent oophorectomy alone without any adjuvant chemotherapy but remained in complete remission [12]. The durations of postoperative follow-up ranged from 4 months to 14 months. The remainder two patients do not have any follow-up information [13].

The key features of concurrent primary ovarian lymphoma and MCT include a wide age range at diagnosis (ranging from 24 years to 68 year of age), minimal involvement by the lymphoid infiltrate, and localized disease (Table 1). Although the prognosis is excellent, the number of cases does not allow to comment on best treatment with respect to simple resection or resection with postoperative adjuvant chemotherapy. In addition, most cases have a short follow-up duration, ranging from 4 to 14 months and, as such, may not reflect the true clinical progression of the disease. Irrespectively, primary ovarian NHL has an excellent prognosis, particularly when diagnosed at early stage [4].

The exclusive presence of follicular lymphoma within the teratoma suggests it may have arisen from it. Hematolymphoid disease has previously been demonstrated to occur within germ cell tumors, especially in the mediastinum. Both myeloid neoplasia and histiocytic sarcoma have most frequently been demonstrated [14]. The detection of immature myeloid precursors in the germ cell tumor has been suggested as the cells of origin for these neoplasms [15]. 


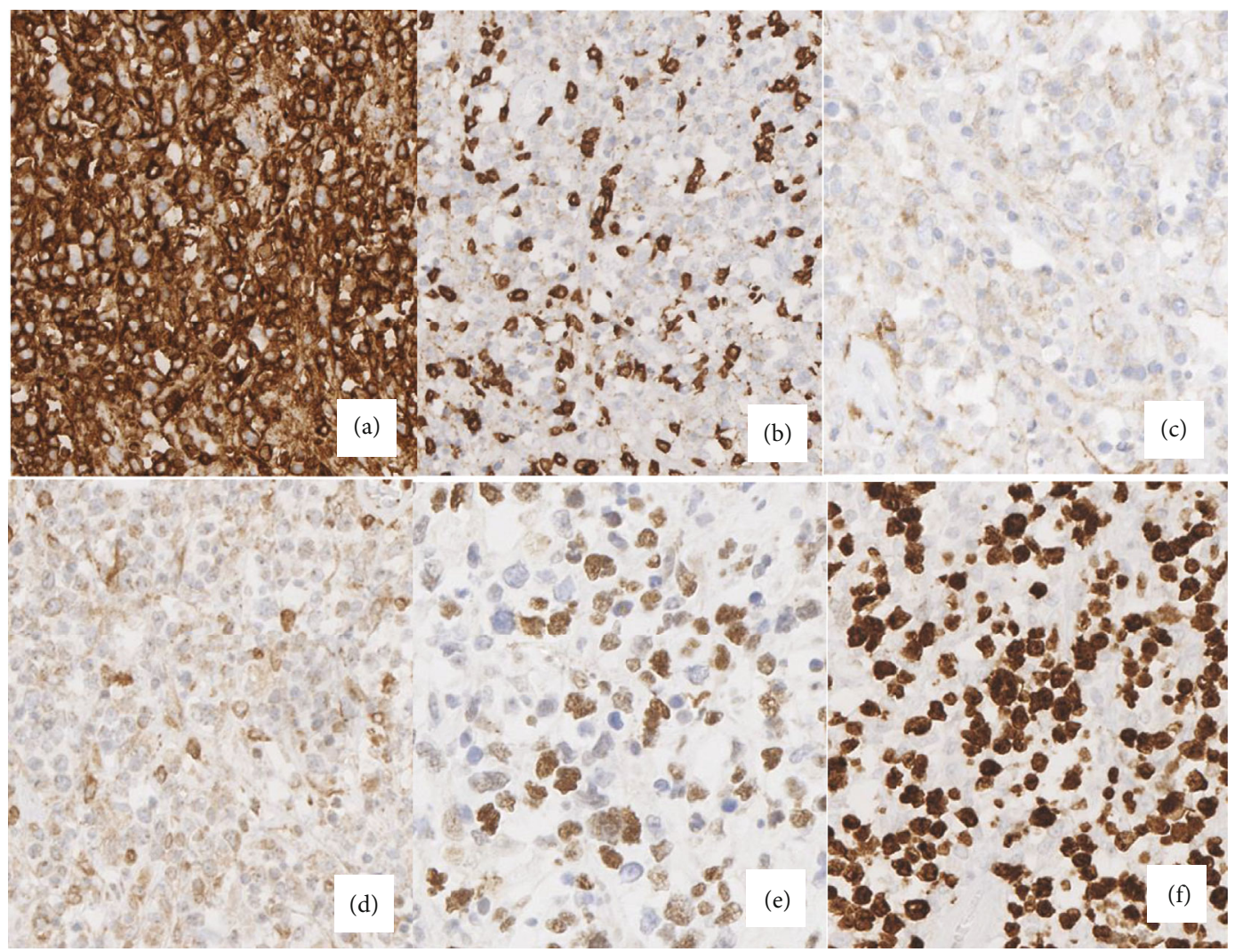

FIGURE 2: Immunohistochemical stains (400x) show that the atypical lymphocytes are positive for CD20 (a), negative for CD3 (b), weakly positive for CD10 (c), weakly positive for BCL2 (d), and positive for BCL 6 (e), with increased Ki-67 proliferation index at $80 \%$ (f).

\section{Conflicts of Interest}

The authors declare that they have no conflicts of interest.

\section{References}

[1] WHO Classification of Tumours Editorial Board, WHO Classification of Tumours of Female Genital Tumors, vol. 4, IARC (International Agency for Research on Cancer), Lyon, 5th edition edition, 2020.

[2] P. B. Clement, J. N. Stall, and R. H. Young, Atlas of Gynecologic Surgical Pathology, Elsevier, Edinburgh, 4th edition edition, 2020.

[3] V. Monterroso, E. S. Jaffe, M. J. Merino, and L. J. Medeiros, "Malignant lymphomas involving the ovary. A clinicopathologic analysis of 39 cases," The American Journal of Surgical Pathology, vol. 17, no. 2, pp. 154-170, 1993.

[4] M. A. Dimopoulos, D. Daliani, W. Pugh, D. Gershenson, F. Cabanillas, and A. H. Sarris, "Primary ovarian non-Hodgkin's lymphoma: outcome after treatment with combination chemotherapy," Gynecologic Oncology, vol. 64, no. 3, pp. 446-450, 1997.

[5] T. Hirakawa, M. Tsuneyoshi, and M. Enjoji, "Squamous cell carcinoma arising in mature cystic teratoma of the ovary: clinicopathologic and topographic analysis," The American Journal of Surgical Pathology, vol. 13, no. 5, pp. 397-405, 1989.

[6] A. McKelvey, D. McKenna, D. McManus, and M. Joyce, "A case of lymphoma occurring in an ovarian teratoma," Gynecologic Oncology, vol. 90, no. 2, pp. 474-477, 2003.
[7] S. Afzal and S. Zaman, "High grade B- cell non- Hodgkin's lymphoma arising in a mature cystic teratoma of the ovary: a case report," International Journal of Molecular and Cellular Medicine, vol. 6, no. 4, pp. 239-242, 2017.

[8] A. Maguire, G. Castriciano, J. Walker et al., "Case study: diffuse large B-cell lymphoma arising in ovarian mature cystic teratoma.," International Journal of Gynecological Pathology, vol. 34, no. 5, pp. 459-464, 2015.

[9] N. Gandhi, I. N. Soomro, S. O'Connor, and V. Sovani, "Primary lymphoma arising in a mature cystic teratoma of the ovary," Histopathology, vol. 61, no. 6, pp. 12381240, 2012

[10] R. Valli, E. Froio, M. I. Alvarez de Celis, V. D. Mandato, and S. Piana, "Diffuse large B-cell lymphoma occurring in an ovarian cystic teratoma: expanding the spectrum of large B-cell lymphoma associated with chronic inflammation," Human Pathology, vol. 45, no. 12, pp. 2507-2511, 2014.

[11] K. Yorita, Y. Tanaka, K. Hirano et al., "Fibrin-associated diffuse large B-cell lymphoma arising in a mature cystic teratoma: a case report," Pathology International, vol. 69, no. 5, pp. 312$314,2019$.

[12] D. B. Seifer, L. M. Weiss, and R. L. Kempson, "Malignant lymphoma arising within thyroid tissue in a mature cystic teratoma," Cancer, vol. 58, no. 11, pp. 2459-2461, 1986.

[13] G. M. Bonneville, R. P. Michel, S. Krishnamurthy, and F. W. Mansour, "Non-Hodgkin lymphomas in mature cystic teratomas: a case report and review of the literature," Journal of Obstetrics and Gynaecology Canada, vol. 39, no. 12, pp. 1171-1175, 2017. 
[14] C. R. Nichols, B. J. Roth, N. Heerema, J. Griep, and G. Tricot, "Hematologic neoplasia associated with primary mediastinal germ-cell tumors," New England Journal of Medicine, vol. 322, no. 20, pp. 1425-1429, 1990.

[15] J. T. Hartmann, C. R. Nichols, J. P. Droz et al., "Hematologic disorders associated with primary mediastinal nonseminomatous germ cell tumors," Journal of National Cancer Institute, vol. 92, no. 1, pp. 54-61, 2000. 